\title{
1 Aneuploidy Spectrum Analysis as a Primer for Copy Number Profiling of 2 Cancer Cells
}

3 Ahmed Ibrahim Samir Khali1 ${ }^{1}$, Anupam Chattopadhyay ${ }^{1, *}$, Amartya Sanyal,,*

4

$5{ }^{1}$ School of Computer Science and Engineering, Nanyang Technological University, 50

$6 \quad$ Nanyang Avenue, Singapore 639798.

$7{ }^{2}$ School of Biological Sciences, Nanyang Technological University, 60 Nanyang Drive,

8 Singapore 637551.

9

10 Email:

11 Ahmed Ibrahim Samir Khalil: ahmedibr001@e.ntu.edu.sg

12 Anupam Chattopadhyay: anupam@ntu.edu.sg

13 Amartya Sanyal: asanyal@ntu.edu.sg

14

* corresponding authors

16 Amartya Sanyal, Ph.D.

17 School of Biological Sciences

18 Nanyang Technological University

1960 Nanyang Drive, SBS-05n-22,

20 Singapore 637551

21 Tel: $(+65) 65138270$

22

23 Anupam Chattopadhyay, Ph.D.

24 School of Computer Science and Engineering

25 Nanyang Technological University

2650 Nanyang Avenue N4-02c-105

27 Singapore 639798

28 Tel: (+65) 67906092 


\section{Abstract}

\section{Motivation}

3 Hyperploidy and segmental aneuploidy are hallmarks of cancer cells due to chromosome 4 segregation errors and genomic instability. In such situations, accurate aneuploidy profiling 5 of cancer data is critical for calibration of copy number $(\mathrm{CN})$-detection tools. Additionally, 6 cancer cell populations suffer from different levels of clonal heterogeneity and aneuploidy

7 alterations over time. The degree of heterogeneity adversely affects the segregation of the 8 depth of coverage (DOC) signal into integral $\mathrm{CN}$ states. This, in turn, strongly influences the 9 reliability of this data for ploidy profiling and copy number variation (CNV) analysis.

\section{Results}

11 We developed AStra framework for aneuploidy profiling of cancer data and assessing their 12 suitability for copy number analysis without any prior knowledge of the input sequencing 13 data. AStra estimates the best-fit aneuploidy profile as the spectrum with most genomic 14 segments around integral $\mathrm{CN}$ states. We employ this spectrum to extract the $\mathrm{CN}$-associated 15 features such as the homogeneity score (HS), whole-genome ploidy level, and CN correction 16 factor. The HS measures the percentage of genomic regions around $\mathrm{CN}$ states. It is used as a 17 reliability assessment of sequencing data for downstream aneuploidy profiling and $\mathrm{CNV}$ 18 analysis. We evaluated the accuracy of AStra using 31 low-coverage datasets from 20 cancer 19 cell lines. AStra successfully identified the aneuploidy spectrum of complex cell lines with 20 HS greater than $75 \%$. Benchmarking against nQuire tool showed that AStra is superior in 21 detecting the ploidy level using both low- and high-coverage data. Furthermore, AStra 22 accurately estimated the ploidy of 26/27 strains of MCF7 (hyperploid) cell line which exhibit 23 varied levels of aneuploidy spectrum and heterogeneity. Remarkably, we found that HS is 24 strongly correlated with the doubling time of these strains.

\section{Availability and implementation}

26 AStra is an open source software implemented in Python and is available at

27 https://github.com/AISKhalil/AStra 
1

2 Cancer cells are plagued with microscopic and submicroscopic numerical and structural

4

\section{Introduction}

alterations of chromosomes. Chromosome missegregation and instability manifests in complex genetic makeups of cancer cells. They can simultaneously acquire near-polyploidy state or aneuploidy at whole-chromosome or portions (segments) of chromosomes [1, $\underline{2}]$. As a result, cancer cells frequently have polyploidy or hyperploidy profiles with widespread segmental aneuploidies [3-5]. Analysis of the Mitelman database indicates $86 \%$ of solid tumors and $72 \%$ of blood cancers exhibit whole-chromosome gain or loss, while $26 \%$ and $6 \%$ of them are near-polyploid, respectively [6]. Also, profiles of 47 ovarian cancer cell lines from the CCLE database showed that up to $74 \%$ of their genomes are altered [7]. Furthermore, cancer cell populations are genetically highly heterogeneous. Sometimes they remain stable with aneuploid karyotype, and in other times, they undergo continuous genetic alterations over passaging time and culture conditions []. Same cell line cultured in different labs exhibits karyogram changes [9]. These facts suggest that cancer cells undergo an adaptive and evolutionary process even under controlled culture environment to generate clonal variability at copy number, gene expression and phenotypic levels [10]. Taken together, all these alterations give rise to 'mosaic' karyotypes of cancer cell populations.

Ploidy information is associated with cancer progression and their varied sensitivity to chemotherapy. Aneuploidy, in general, is considered as a marker of poor prognosis and their presence correlates with chemoresistance [11]. In fact, antiproliferative compounds targeting aneuploid cells serve as a new chemotherapeutic strategy for cancer cells [12]. Furthermore, characterization of the aneuploidy profile forms the basis of $\mathrm{CNV}$ detection for single-sample cancer data[13]. Most read depth (RD)-based tools use the global RD median for setting the $\mathrm{CN}$ reference $(2 \mathrm{~N})[\underline{14}, \underline{15}]$ or the ploidy states $[\underline{16}, \underline{17}]$. However, the global median may not accurately approximate any of the ploidy states, especially in case of hyperploid cancer cells. Moreover, some of these tools require the information of $\mathrm{CN}$ gain/loss percentage for better modeling of the RD signal. Therefore, it is necessary to estimate the overwhelming extent of aneuploidy spectrum for calibrating the CNV detection tools.

The chromosomal aneuploidy and microscopic-level segmental gain or loss are classically analyzed by karyotyping. Deviation from 'normal' karyotype is a common hallmark of cancer cells $[\underline{18}, \underline{19}]$. Whole-genome ploidy level with modal chromosome number has been routinely characterized for most cancer cell lines using imaging techniques by individual labs and cell repositories. However, cancer cells undergo clonal evolution over time and therefore 
34 ploidy characterization may not be stable and reliable. Moreover, karyotyping is a lowresolution technique. Therefore, standard karyotype cannot distinguish between two genomes with normal chromosome complement but harboring different submicroscopic chromosomal aneuploidies. Taken together, the dynamics of these microscopic and submicroscopic alterations represents timestamp 'barcode' of aneuploidy spectrum of cancer cells. A rough estimation of this spectrum can form the background knowledge needed for accurate downstream sequence analysis of cancer cell line.

The advent of array- and next-generation sequencing (NGS)- based techniques allowed higher-resolution estimation of the complete repertoire of aneuploidy at different structural levels. Most genome-level ploidy tools compute ploidy levels by assessing the distribution of allele frequency at biallelic single-nucleotide polymorphisms (SNPs) [20-22]. These SNPbased tools require very high-coverage sequencing data. As a result, they were successfully applied to small genomes like yeast but are less adaptable in human scenario, especially for low-coverage sequencing data. Additionally, PloidyNGS estimates ploidy by assuming different ratios of biallelic SNPs at different ploidy levels. However, this can bring ambiguity in defining the diploid versus tetraploid with 0.5/0.5 ratios. Recently, nQuire was developed to estimate ploidy by fitting the coverage signal to pre-determined frequency distributions with fixed contribution from each ploidy state [23]. Nevertheless, nQuire cannot capture the hyperploidy profiles which have variable combinations of different ploidy levels. Hence, it is necessary to capture the contribution of each ploidy level into the complete aneuploidy profile (aneuploidy spectrum) without any pre-defined assumption of whole-genome ploidy. Additionally, ploidy tools are implicitly built on a fundamental assumption that cancer cell lines are clonal in origin, and therefore, they are 'homogeneous' in nature. The pervasive presence of cellular variability and genetic heterogeneity in cancer cells [24] necessitate estimating the homogeneity level in cancer population as a condition for accurate ploidy estimation.

Here, we introduce AStra (Aneuploidy Spectrum (detection) through read depth analysis), a Python-based software for capturing the complete aneuploidy profile of cancer genome without an explicit assumption of the ploidy level of the input sequencing data. AStra is based on the fundamental reasoning that most genomic segments belong to integral $\mathrm{CN}$ states. AStra scans the RD signal to find the $\mathrm{CN}$ reference that best segregates genomic segments into distinct copy-number states to estimate the aneuploidy spectrum. We employ this spectrum to compute several $\mathrm{CN}$-associated attributes. The homogeneity score measures 
67 the reliability of the input cancer data for downstream aneuploidy and CNV analysis. The 68 tool also infers the whole-genome ploidy level, the $\mathrm{CN}$ correction factor and $\mathrm{CN}$ gain/loss 69 percentage for calibrating CNV detection tools. To sum up, AStra provides the prerequisite 70 knowledge of the copy number-associated features for the DOC-based analysis of cancer 71 cells. 


\section{2. Methods}

\section{$2 \quad 2.1$ AStra framework}

3 AStra utilizes the RD signal of the input data to define its aneuploidy spectrum and to

4 compute its important features for accurate ploidy and $\mathrm{CNV}$ analysis. The main key for

5 defining the aneuploidy spectrum is the accurate estimation of $\mathrm{CN}$ reference $(2 \mathrm{~N})$ by

6 scanning the RD signal. $\mathrm{CN}$ reference should be defined as the RD value that segregates $\mathrm{RD}$

7 signal distribution into segments of integral CN states. In our pipeline, NGS reads are used

8 first to compute the RD signal. This RD signal is clustered using Pruned Exact Linear Time

9 (PELT) method [25] into primary segments of different RD values. Then, we employ 10

10 different unimodal/multimodal distributions to define the candidate $\mathrm{CN}$ references and the

11 associated $\mathrm{CN}$ states (Fig. 1a). Under each candidate reference, we merge the adjacent

12 primary segments with same $\mathrm{CN}$ state into large contiguous segment to generate the

13 aneuploidy profile. Then, we compute the Centralization Error (CE) of that aneuploidy

14 profile as the weighted summation of differences between the estimated $\mathrm{CN}$ of segments and 15 their CN states (Fig. 1b). The correct $\mathrm{CN}$ reference is the one that achieves the minimum CE.

16 Once the $\mathrm{CN}$ reference is settled, we compute the ploidy spectrum as the percentages of each

17 ploidy level into the complete aneuploidy profile. Finally, the homogeneity score (HS) is calculated as the percentage of genomic segments with estimated $\mathrm{CN}$ around their integral CN states (Fig. 1c). We also define the whole-genome ploidy ('diploid,' 'triploid,' or 'tetraploid') and determine the validity of usage of the global median (RD signal) as $\mathrm{CN}$

21 reference.

\subsubsection{RD frequency distribution}

Cancer cells exhibit multimodal distribution as a combination of the underlying unimodal distributions at different $\mathrm{CN}$ states. Therefore, we build $10 \mathrm{RD}$ frequency distributions as weighted summation of Gaussian/Normal distributions centralized at different $\mathrm{CN}$ states to compute the candidate $\mathrm{CN}$ reference (Fig. 1a):

$$
f(x)=\sum_{i=2}^{6} c * w_{i} *\left(\frac{1}{\sqrt{\left(2 \pi \sigma^{2}\right)}} * e^{-\frac{(x-i)^{2}}{2 \sigma^{2}}}\right), x \geq 0
$$

such as

$$
\int_{0}^{\infty} f(x) \cdot d x=1
$$




$$
\int_{0}^{\infty} \sum_{i=2}^{6} c * w_{i} *\left(\frac{1}{\sqrt{\left(2 \pi \sigma^{2}\right)}} * e^{-\frac{(x-i)^{2}}{2 \sigma^{2}}}\right) \cdot d x=1
$$

$$
\sum_{i=2}^{6} c * w_{i} * \int_{0}^{\infty}\left(\frac{1}{\sqrt{\left(2 \pi \sigma^{2}\right)}} * e^{-\frac{(x-i)^{2}}{2 \sigma^{2}}}\right) \cdot d x=1
$$

$$
\sum_{i=2}^{6} c * w_{i}=1
$$

$$
c=\frac{1}{\sum_{i=2}^{6} w_{i}}
$$

where $i$ is the copy-number state $(2,3,4,5,6), w_{i}$ is the weight of the Gaussian distribution at state $i(0,1,0.5,0.25,0.125,0.0625)$ and $c$ is a constant for normalization of the probability distribution function. The standard deviation $(\sigma)$ is $0.5 / 3$ to make $f(x)=0$ at the boundaries of copy-number intervals $(1.5,2.5,3.5, \ldots)$.

\subsubsection{Estimation of candidate $\mathrm{CN}$ reference}

For each of the $10 \mathrm{RD}$ frequency distributions, candidate $\mathrm{CN}$ reference is defined as the $\mathrm{RD}$ spaced $\mathrm{RD}$ values. At each $\mathrm{RD}$ value $k$, a multimodal distribution $f_{k}(x)$ is generated assuming this is the copy number reference $(2 \mathrm{~N})$. And, a rank $R_{k}$ is computed as the convolution between $r(x)$ and $f_{k}(x)$ :

$$
R_{k}=\sum_{j=s}^{e} r(j) * f_{k}(j), \quad k \in[s, e]
$$

Finally, $\mathrm{RD}$ value $k$ with the maximum $\operatorname{rank} R_{k}$ is chosen as the candidate $\mathrm{CN}$ reference.

\subsubsection{Centralization error}

Given the candidate $\mathrm{CN}$ reference, we merge the genomic segments to divide the genome into contiguous segments of distinct $\mathrm{CN}$ states to form the chromosomal aneuploidy profile. Under the correct $\mathrm{CN}$ reference, most of these segments should have $\mathrm{CN}$ around integral $\mathrm{CN}$ states. CE measures the degree of localization of these segments near CN states (Fig. 1b):

$$
C N_{i}=R D_{i} * \frac{2}{C N R}
$$

$$
S_{i}=R N E\left(C N_{i}\right)
$$

$$
C E=\sum_{j=1}^{n} C E_{j}
$$


56 where $i$ is the segment index, $j$ is the $\mathrm{CN}$ state/interval, $R D_{i}$ is the median RD per segment,

$57 C N_{i}$ is the copy number of the segment, CNR is the candidate $\mathrm{CN}$ reference, $S_{i}$ is the $\mathrm{CN}$

58 state of the segment (round-to-nearest value of $C N_{i}$ ), $W_{i}$ is the width of the segment $i$, and

$59 C E_{j}$ is the centralization error of segments of $\mathrm{CN}$ state $j$.

\section{$60 \quad$ 2.1.4 Characterization of the aneuploidy spectrum}

61 The aneuploidy profile, with minimum CE, is decoded into the aneuploidy spectrum which

62 contains the percentage of genome segments in each $\mathrm{CN}$ state/interval. HS measures the

63 degree of localization of genomic segments into integral CN states. HS is computed as the

64 percentage of segments that are around the integral CN states (Fig. 1c):

$$
H S=\frac{\sum_{i} W_{i}, \quad\left|S_{i}-C N_{i}\right| \leq 0.25}{\sum_{i} W_{i}}
$$

66 where $i$ is the segment index, $C N_{i}$ is the copy number of the segment, $S_{i}$ is the CN state of the

67 segment, and $W_{i}$ is the width of the segment $i$. Sequencing data with high HS are suitable for $68 \mathrm{CNV}$ and ploidy analysis. Finally, we infer the ploidy level ('diploid', 'triploid', 'tetraploid') 69 as the $\mathrm{CN}$ state harboring maximum percentage of genomic segments. Also, we compute the 70 'CN correction factor' as the RD signal ratio between the global median and the ploidy state. 71 If any $\mathrm{CNV}$ tool computes $\mathrm{CN}$ reference solely based on the global median, this correction 72 factor can be used to correct $\mathrm{CN}$ reference and copy number estimates of genomic segments. 
1

2

3

4

\section{Results}

\subsection{Coverage is not the only criteria for accurate aneuploidy profiling and copy number analysis of cancer cells}

Cancer cells suffer from a degree of hyperploidy and segmental aneuploidy due to chromosomal instability. This results in a genome which contains a collection of genomic segments with different $\mathrm{CN}$ states. In such a situation, the copy number profiling can still be performed using appropriate modeling of RD signal by a multimodal distribution if and only if the $\mathrm{CN}$ profiles are well separated into integral $\mathrm{CN}$ states. However, this condition depends on the other source of biases in cancer sequencing data. The main bias originates from the purity of the sample, clonal variations, and heterogeneity of the cancer cells/samples which adversely affects peak separation. In addition, coverage signal of cancer cells may be affected by wave artifacts which may contribute to signal variations and peak separation [26]. In low coverage data, the effects of heterogeneity and wave artifacts are magnified due to the sparseness of reads representing each region of the genome. Consequently, it is a prerequisite to determine the suitability of a sequencing data for downstream copy number or ploidy analyses based on its coverage and heterogeneity level. Therefore, we introduced the homogeneity score (HS) to evaluate the degree of separation between different copy number states (peaks) of the cancer data.

We analyzed low-coverage $(<3 \mathrm{x})$ sequencing data of 20 cancer cell line data with a total of 31 replicates (Supplementary Table 1). We found that HS is weakly correlated with the coverage of the data (Spearman correlation $=-0.3104$, p-value $=0.0658$ ) (Fig. 2a). Interestingly, we found that HS varies considerably among biological replicates from the same laboratory as well as among the same cell line sequencing data from different studies. For example, biological replicates (denoted with A1 and A2 suffices) from same study exhibit different HS as evidenced from PC3 (78.86\%, 71.9\%), MCF7 (92.08\%, 72.47\%), MDA-MB-231 (89.81\%, 74.48\%), MDA-MB-468 (90.37\%, 77.39\%) and SK-BR-3 (75.45\%, $69.76 \%$ ). These differences may be partially explained by coverage effect since merging both replicates (denoted with A suffix) slightly improve the HS in the case of PC3 (78.34\%), SKBR-3 (77.54\%) and MDA-MBA-468 (92.47\%). However, replicate-merging decreases HS in case of MCF7 (66.27\%) and MDA-MB-231 (73.07\%), suggesting coverage may not be the only key for separating $\mathrm{CN}$ profiles into integral $\mathrm{CN}$ states. In order to study the relationship between HS and data coverage, we subsampled high-coverage A427 cancer data and found 
33 that homogeneity scores remain same for all A427 subsamples (10x to 0.3x) with a maximum 34 change of $2 \%$ (Supplementary Table 2). Also, HS is not affected by the whole genome ploidy 35 level (Fig. 2b). Therefore, we concluded that coverage cannot be used as an absolute threshold to assess the suitability of cancer data for copy number analysis. We found that sequencing data with higher HS are less affected by the biases of cancer genomes. After examining all the datasets, we concluded that a minimum homogeneity score of $75 \%$ provides an empirical threshold that separates RD frequency distributions peaks and centralized them around CN states. For example, A375_A, A375_B, MDA-MB-468_B, PC3_B, T47D_A, and K562_B data showed low HS and low separation of RD distribution peaks (Supplementary Fig. 1). Out of the 36 samples, aneuploidy profiles of 22 samples with HS $>75$ are correctly identified based on the detection of multimodal peaks and known karyotype. The other 14 samples with HS $\leq 75$ have 6 accurate and 8 wrong aneuploidy profiles. In such a case, we recommend checking the aneuploidy spectrum manually. Therefore, we argue that homogeneity scoring should be a prerequisite test to be performed on every sequencing data before downstream $\mathrm{CNV}$ and aneuploidy analyses.

\subsection{AStra assumption captures the complex aneuploidy spectrum of cancer cells}

Current RD-based ploidy estimation tools compute the whole-genome aneuploidy profile based on best fitting to the empirical models under each ploidy assumptions (diploid, triploid, tetraploid, etc.). However, cancer cells harbor different degrees of hyperploidy that cannot be captured in model with specific ratios of different ploidy states. In contrast, AStra is built on a fundamental assumption that coverage signal of the cancer genome exhibits multimodal distribution with most genomic segments distributed around the integral copy number states. For that, AStra examines many multimodal distributions with different weights of CN states to define the candidate $\mathrm{CN}$ reference. $\mathrm{CN}$ reference is chosen based on the model that best assimilates the genomic segments (and not RD fitting) into distinct peaks of multimodal distribution. This allows us to apply AStra on low-coverage data and ignore the adverse effects of signal dispersion.

60 We applied AStra for 36 samples of low-coverage cancer cells, A427 high-coverage cancer 61 cell, and 3 high-coverage normal cells (Supplementary Table 1). AStra computes the aneuploidy profile of low-coverage $(<3 \mathrm{x})$ data in less than 3 minutes and high-coverage $(28 \mathrm{x})$ in about 15 minutes (Supplementary Table 1). Astra successfully identified the accurate aneuploidy spectrum of all cell lines, with HS greater than $75 \%$, based on two conditions.

65 First, copy number reference and higher $\mathrm{CN}$ states are detected as peaks of multimodal 
66 distribution (Fig. 2b, Supplementary Fig. 2). Second, the major peak confirms the known

67 karyotype for the ploidy cells (Supplementary Table 1). Similarly, the two major peaks confirm the known karyotype for the hyperploidy cells (Supplementary Table 1). For example, AStra detected the location four peaks $(1 \mathrm{~N}, 2 \mathrm{~N}, 3 \mathrm{~N}, 4 \mathrm{~N})$ accurately of $\mathrm{VCaP}$ at both $10 \mathrm{~Kb}$ and $100 \mathrm{~Kb}$ bins (Fig. $2 \mathrm{~b}$ ). Although the RD-frequency of $\mathrm{VCaP}$ at $10 \mathrm{~Kb}$ can be wrongly interpreted as unimodal distribution, AStra still can identify the accurate values of $\mathrm{CN}$ states.

We then analyzed the performance of our 10 empirical models for estimating the aneuploidy spectrum by comparing their CEs on different datasets. We also counted the number of datasets that has minimum CE using each model. We found that 9 out of the 10 models results in the minimum $\mathrm{CE}$ for at least one dataset (Fig. 2c). M4, M10, and M1 show the best performance as they gave the minimum CE for 5, 5 and 4 datasets, respectively. Remarkably, there is at least one additional model that results in similar CE $(<5 \%$ difference) for each dataset (Fig. 2d). Therefore, it confirms our hypothesis that aneuploidy spectrum of cancer cells cannot be comprehended based on a single empirical model for each ploidy level. As a conclusion, our models allow AStra to detect the aneuploidy profiles of hyperploidy cancer cells.

\subsection{AStra best estimates the whole-genome ploidy in low-coverage WGS data}

We have also benchmarked our tool against nQuire by applying it on 10 cancer cell lines with known karyotype information and 3 normal cell lines (Supplementary Table 3). Although most cancer cell lines have a hyperploidy profile, nQuire employ models for diploid, triploid, and tetraploid only. Therefore, for a fair comparison, we keep only cancer cell lines which have a major peak at the ploidy $\mathrm{CN}$ state and a ratio between genomic segments at major $\mathrm{CN}$ state and at any other states is at least 2. This resulted in 6 diploid, 2 triploid and 2 tetraploid cell lines. We found that nQuire estimate the ploidy level of all cells as tetraploid (Supplementary Table 3). In comparison, AStra correctly estimates the nearest whole-genome ploidy level based on the $\mathrm{CN}$ state with the highest percentage of genomic segments 93 (Supplementary Table 3). Although both tools utilize multimodal distribution for estimating the whole-genome aneuploidy, AStra performed better than nQuire. This may be attributed to the fact that Astra evaluates the multimodal fitting based on the genomic segments, not based on the RD signal. This allows Astra to compute the copy number states even at low-coverage data. Also, nQuire 
99 aneuploidy change. Similarly, nQuire triploid distribution assumes that the data distribution 100 can have only two peaks $2 \mathrm{~N}$ and $3 \mathrm{~N}$, and so on. In contrast, AStra models account for any percentage of segmental aneuploidy, and therefore they can capture the complexity of hyperdiploid, near-triploid, or higher ploidy levels.

\subsection{Aneuploidy spectrum is critical for calibration of CNV detection tools}

Most CNV detection tools are built on some basic assumptions or user-defined knowledge of ploidy and percentage of copy number gain/loss for accurate modeling of RD signal data. For example, FREEC uses the whole-genome ploidy level to define the $\mathrm{CN}$ state [16] while ReadDepth [15] uses the percentage of gain/loss to adjust their underlying Poisson/negative binomial distribution. Similarly, CNVnator [14] assumes that $99 \%$ of the genome is CNV free. However, this information changes across cell lines and between same cell line data obtained from different labs owing to clonal variability as presented in this study (Supplementary Table 1).

The global RD median can be correctly used as ploidy CN state if we assume that the copy number gains and losses are similar. However, this is not the case for most cancer cells. For example, diploid cell lines (CHP-212, IMR-32, HepG2, CAL-51, SUM159) have different percentage of gain (loss) ranging from $1.4 \%(0 \%)$ to $18.11 \%(3 \%)$. Therefore, we compute the median error as the relative shift of the global median $(\mathrm{g})$ to the nearest $\mathrm{CN}$ state $(\mathrm{s})$

$\left(\frac{|g-s|}{s}\right)$. This error can be used to check the validity of ploidy assumption (Fig. 3a). If this error is large (Fig. 3b), the only solution is to correct the copy number of the CNV regions. This can be achieved by computing $\mathrm{CN}$ correction factor as the ratio between ploidy state and the global median (Supplementary Table 1). User can use this ratio to adjust the CN of output segments of the tool. Based on these observations, it can be concluded that knowledge of aneuploidy spectrum is fundamental for selecting and calibrating CNV detection tools. Aneuploidy has been linked with cell proliferation defects and lethality in almost all organisms from yeast to human $[\underline{27}, \underline{28}]$. In mouse and human, chromosomal aneuploidy, with exceptions of few chromosomes, leads to embryonic lethality and the viable trisomies display severe abnormalities [29-31]. Contrasting to its adverse effect on cell proliferation, aneuploidy is associated with uncontrolled cellular growth and cancer [32]. Majority of all human solid tumors carry numerical changes in karyotype [ㅍ3]. Mouse models of 
$131[\underline{34}, \underline{35}]$. In order to understand the effect of aneuploidy profile change on the phenotype of 132 cancer cells, we revisited the study of 27 strains of MCF7 cell lines to obtain the sequencing 133 data [10].

134 We applied AStra on all the 27 strains of MCF7 collected from different labs (Supplementary

135 Table 4). AStra estimated the aneuploidy spectrum accurately for 26/27 strains with the 136 majority of segments with $\mathrm{CN}$ states of $3 \mathrm{~N}$ or $4 \mathrm{~N}$. This confirms that MCF7 karyotype is 137 hypertriploid to hypotetraploid [36]. Out of 27, only the spectrum of strain Z is wrongly 138 identified since it has peaks around the boundaries of $\mathrm{CN}$ intervals $(2.5 \mathrm{~N}$ and $3.5 \mathrm{~N})$. 139 Interestingly, we also found that strain Z has HS of $65 \%$ which is lower than our empirically 140 estimated threshold of $>75 \%$. The ploidy levels of the rest 26 strains are inferred as 7 triploid 141 and 19 tetraploid based on the $\mathrm{CN}$ state with the maximum percentage of genomic segments 142 (Supplementary Fig. 3). This confirms the idea that populations of the same cell line may 143 have different ploidy profiles based on the culture condition and passage number. Moreover, 144 we found that HS varies greatly among the 26 strains from $56 \%$ to $96 \%$ with only 14 strains 145 achieving our cutoff threshold of $>75 \%$ (Fig. 3c). The other 12 strains with low HS suffer 146 from a higher degree of heterogeneity. The RD signal of these heterogeneous strains shows a 147 strong effect of wave artifacts and dispersion (Supplementary Fig. 3d-e). Remarkably, we 148 found that the homogeneity score is correlated with the doubling time (Spearman correlation $149=0.7718, \mathrm{p}$-value $=2.4215 \mathrm{e}-06)($ Fig. 3d) reported earlier for these strains [10]. Additionally, 15011 strains (viz. A, F, R, S, T, U, V, W, X, Y, and Z) form one group of similar HS and data 151 coverage (Fig. 3c). These same strains were grouped under one cluster in the earlier study 152 based on their SNV (single nucleotide variant) cellular prevalence [10]. This suggests that 153 homogeneity score itself is informative beside the aneuploidy spectrum information. 


\section{4. Discussion}

2 The genetic profiling of cancer cells is complex. The alterations at the numerical and 3 structural levels and at multitude of length-scales are difficult to detect using a single 4 approach. At the highest level, karyotype alterations include the whole-genome ploidy, 5 chromosomal aneuploidy, and segmental gain/loss. These alterations can limit the accurate 6 estimation of the copy number reference by CNV detection tools. Some CNV detection tools 7 require the prior knowledge of ploidy level while other tools rely on the global median of the 8 coverage signal for estimation of $\mathrm{CN}$ reference. The latter can be misleading in most cancer 9 cells. Therefore, we have developed AStra, a Python-based tool, which can segregate the 10 genome into segments of integral $\mathrm{CN}$ states. This allows AStra to model the complex

11 hyperploid cancer cells in addition to polyploid cells without any prior knowledge of the 12 input cell line.

13 Additionally, the heterogeneity of cancer cells adds to another level of complexity. The level 14 of heterogeneity can restrict the legible use of cancer data for aneuploidy profiling. None of 15 the existing ploidy estimation tools provide any quality measurement of the input data. Data 16 coverage is an important factor that affects the quality of the data. However, we showed that 17 coverage cannot be solely relied on as a cut-off threshold. To overcome this problem, AStra 18 defines the homogeneity score (HS) which measures the impact of heterogeneity along with 19 other systematic biases in the cancer data. Our comprehensive analyses of cancer cell lines 20 and visual inspection of the results suggest that a minimum homogeneity score of $75 \%$ can be 21 used as a confidence threshold for downstream estimation of ploidy profile.

22 In conclusion, we recommend using AStra results as a quick reference guide to assess the 23 suitability of cancer NGS data for copy number analysis and to calibrate the parameters of 24 CNV detection tools. 


\section{Declarations}

\section{Acknowledgment}

3 We acknowledge Sanyal and Chattopadhyay lab members for their valuable comments.

\section{$4 \quad$ Funding}

5 This work was supported by Nanyang Technological University's Nanyang Assistant

6 Professorship grant and Singapore Ministry of Education Academic Research Fund Tier 1

7 grant to AS. AC is supported by Nanyang Technological University Start-up grant.

\section{Author's contributions}

9 AS, AC and AISK conceived the project. AISK developed AStra software with inputs from

$10 \mathrm{AS}$ and $\mathrm{AC}$ and performed all the analyses. AS, AISK and AC analyzed the data and

11 prepared the manuscript. All authors read and approved the final manuscript.

\section{Ethics approval and consent to participate}

13 Not applicable.

\section{Competing interests}

15 The authors declare that they have no competing interests. 
3

4

\section{References}

1. Sansregret, L. and C. Swanton, The Role of Aneuploidy in Cancer Evolution. Cold Spring Harb Perspect Med, 2017. 7(1).

2. Gordon, D.J., B. Resio, and D. Pellman, Causes and consequences of aneuploidy in cancer. Nat Rev Genet, 2012. 13(3): p. 189-203.

3. Speicher, M.R., et al., Analysis of chromosomal alterations in non-small cell lung cancer by multiplex-FISH, comparative genomic hybridization, and multicolor bar coding. Lab Invest, 2000. 80(7): p. 1031-41.

4. Schulze, S. and I. Petersen, Gender and ploidy in cancer survival. Cell Oncol (Dordr), 2011. 34(3): p. 199-208.

5. Lazarevic, V., et al., Prognostic significance of high hyperdiploid and triploid/tetraploid adult acute myeloid leukemia. Am J Hematol, 2015. 90(9): p. 8005.

6. Zasadil, L.M., E.M. Britigan, and B.A. Weaver, $2 n$ or not $2 n$ : Aneuploidy, polyploidy and chromosomal instability in primary and tumor cells. Semin Cell Dev Biol, 2013. 24(4): p. 370-9.

7. $\quad$ Domcke, S., et al., Evaluating cell lines as tumour models by comparison of genomic profiles. Nat Commun, 2013. 4: p. 2126.

8. Fisher, R., L. Pusztai, and C. Swanton, Cancer heterogeneity: implications for targeted therapeutics. Br J Cancer, 2013. 108(3): p. 479-85.

9. Thompson, S.L. and D.A. Compton, Chromosomes and cancer cells. Chromosome Res, 2011. 19(3): p. 433-44.

10. Ben-David, U., et al., Genetic and transcriptional evolution alters cancer cell line drug response. Nature, 2018. 560(7718): p. 325-330.

11. Li, R., et al., Chromosomal alterations cause the high rates and wide ranges of drug resistance in cancer cells. Cancer genetics and cytogenetics, 2005. 163(1): p. 44-56.

12. Tang, Y.C., et al., Identification of aneuploidy-selective antiproliferation compounds. Cell, 2011. 144(4): p. 499-512.

13. Alkodsi, A., R. Louhimo, and S. Hautaniemi, Comparative analysis of methods for identifying somatic copy number alterations from deep sequencing data. Brief Bioinform, 2015. 16(2): p. 242-54.

14. Abyzov, A., et al., CNVnator: an approach to discover, genotype, and characterize typical and atypical CNVs from family and population genome sequencing. Genome Res, 2011. 21(6): p. 974-84.

15. Miller, C.A., et al., ReadDepth: a parallel $R$ package for detecting copy number alterations from short sequencing reads. PLoS One, 2011. 6(1): p. e16327.

16. Boeva, V., et al., Control-FREEC: a tool for assessing copy number and allelic content using next-generation sequencing data. Bioinformatics, 2012. 28(3): p. 423-5.

17. Eisfeldt, J., et al., TIDDIT, an efficient and comprehensive structural variant caller for massive parallel sequencing data. F1000Res, 2017. 6: p. 664.

18. Bakhoum, S.F. and C. Swanton, Chromosomal instability, aneuploidy, and cancer. Front Oncol, 2014. 4: p. 161.

19. Giam, M. and G. Rancati, Aneuploidy and chromosomal instability in cancer: $a$ jackpot to chaos. Cell Div, 2015. 10: p. 3.

20. Augusto Correa Dos Santos, R., G.H. Goldman, and D.M. Riano-Pachon, ploidyNGS: visually exploring ploidy with Next Generation Sequencing data. Bioinformatics, 2017. 33(16): p. 2575-2576. 
21. Yu, Z., et al., CLImAT: accurate detection of copy number alteration and loss of heterozygosity in impure and aneuploid tumor samples using whole-genome sequencing data. Bioinformatics, 2014. 30(18): p. 2576-83.

22. Margarido, G.R. and D. Heckerman, ConPADE: genome assembly ploidy estimation from next-generation sequencing data. PLoS Comput Biol, 2015. 11(4): p. e1004229.

23. Weiss, C.L., et al., nQuire: a statistical framework for ploidy estimation using next generation sequencing. BMC Bioinformatics, 2018. 19(1): p. 122.

24. Altschuler, S.J. and L.F. $\mathrm{Wu}$, Cellular heterogeneity: do differences make a difference? Cell, 2010. 141(4): p. 559-63.

25. Killick, R., P. Fearnhead, and I.A. Eckley, Optimal detection of changepoints with a linear computational cost. Journal of the American Statistical Association, 2012. 107(500): p. 1590-1598.

26. van Heesch, S., et al., Systematic biases in DNA copy number originate from isolation procedures. Genome Biol, 2013. 14(4): p. R33.

27. Niwa, O., Y. Tange, and A. Kurabayashi, Growth arrest and chromosome instability in aneuploid yeast. Yeast, 2006. 23(13): p. 937-50.

28. Williams, B.R., et al., Aneuploidy affects proliferation and spontaneous immortalization in mammalian cells. Science, 2008. 322(5902): p. 703-9.

29. Moerman, P., et al., The pathology of trisomy 13 syndrome. A study of 12 cases. Hum Genet, 1988. 80(4): p. 349-56.

30. Lin, H.Y., et al., Clinical characteristics and survival of trisomy 18 in a medical center in Taipei, 1988-2004. Am J Med Genet A, 2006. 140(9): p. 945-51.

31. Antonarakis, S.E., et al., Chromosome 21 and down syndrome: from genomics to pathophysiology. Nat Rev Genet, 2004. 5(10): p. 725-38.

32. Weaver, B.A. and D.W. Cleveland, The aneuploidy paradox in cell growth and tumorigenesis. Cancer Cell, 2008. 14(6): p. 431-3.

33. Albertson, D.G., et al., Chromosome aberrations in solid tumors. Nat Genet, 2003. 34(4): p. 369-76.

34. Weaver, B.A., et al., Aneuploidy acts both oncogenically and as a tumor suppressor. Cancer Cell, 2007. 11(1): p. 25-36.

35. Sotillo, R., et al., Mad2 overexpression promotes aneuploidy and tumorigenesis in mice. Cancer Cell, 2007. 11(1): p. 9-23.

36. Rondon-Lagos, M., et al., Effect of low doses of estradiol and tamoxifen on breast cancer cell karyotypes. Endocr Relat Cancer, 2016. 23(8): p. 635-50. 


\section{Figures}

Fig. 1

AStra framework. (a) Representative multimodal coverage distributions (M1 to M10) which are utilized to detect $\mathrm{CN}$ reference by scanning the input $\mathrm{RD}$ signal distribution. The $\mathrm{RD}$ frequency distribution of A427 cell line is shown as an input example. (b) Illustration to compute centralization error which allows to obtain the best-fit aneuploidy profile. Each blue line represents a genomic segment while the red arrow is the error weight of this segment (the difference between the $\mathrm{CN}$ of that segment and the nearest $\mathrm{CN}$ state). (c) Illustration to calculate the homogeneity score (HS). Each blue line represents a segment with estimated CN within $0.25 \mathrm{~N}$ from the nearest $\mathrm{CN}$ state (homogeneous segment) whereas each red line represents a segment with estimated $\mathrm{CN}>0.25 \mathrm{~N}$ from the nearest $\mathrm{CN}$ state (heterogeneous segment). 


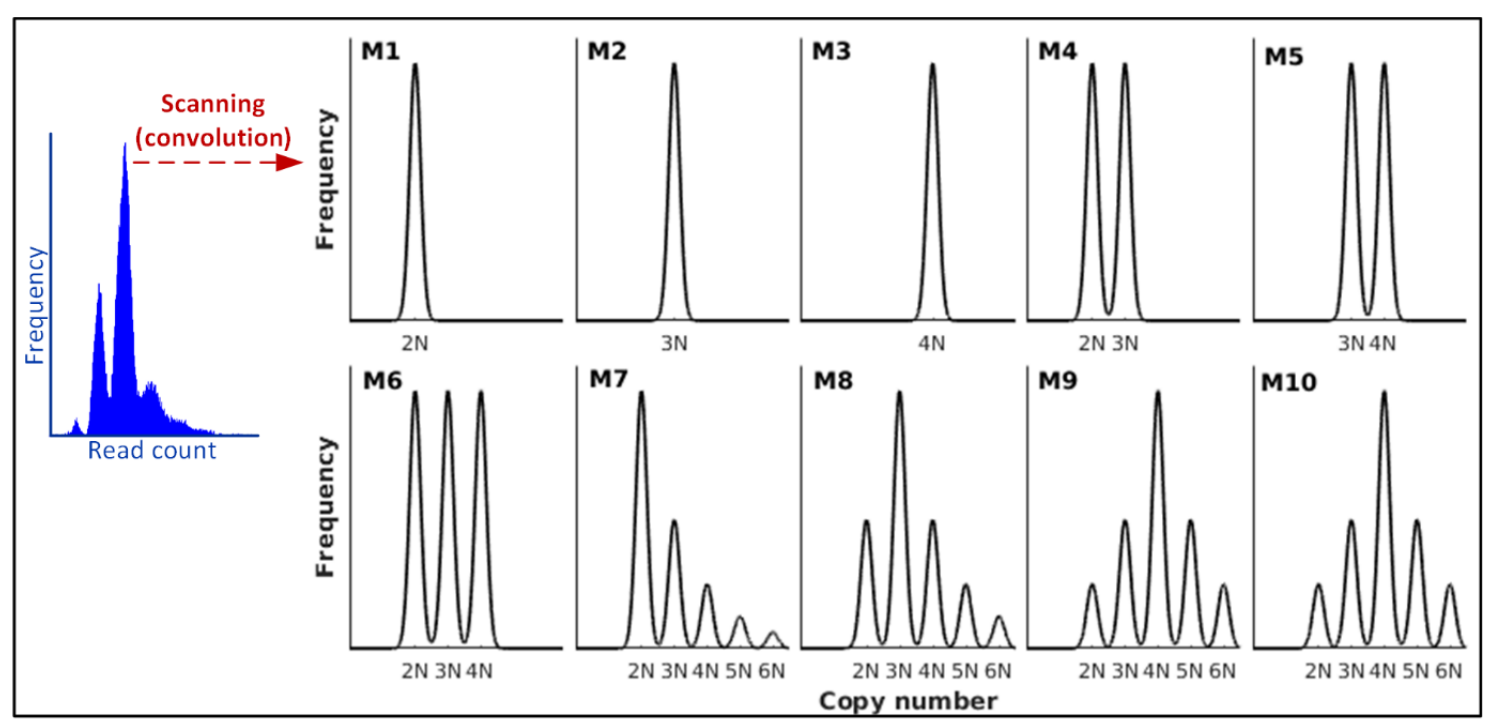

(a) Candidate $\mathrm{CN}$ reference

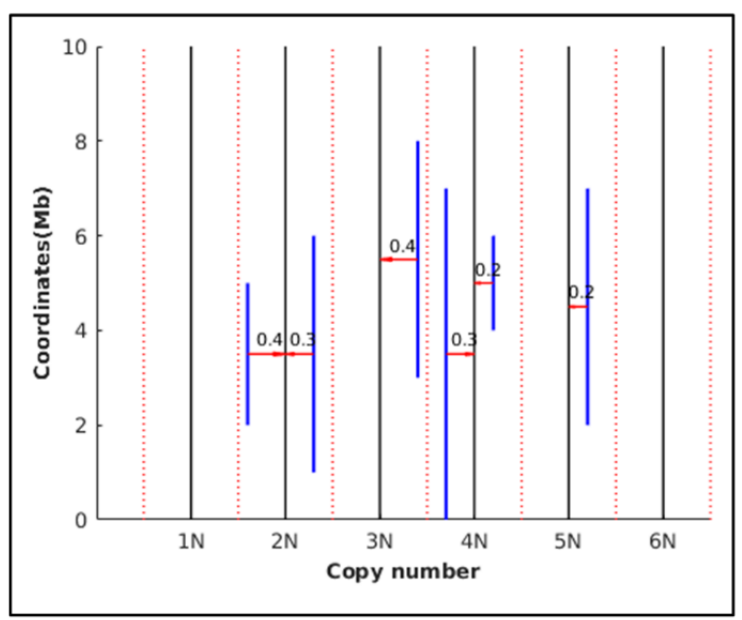

(b) Centralization error

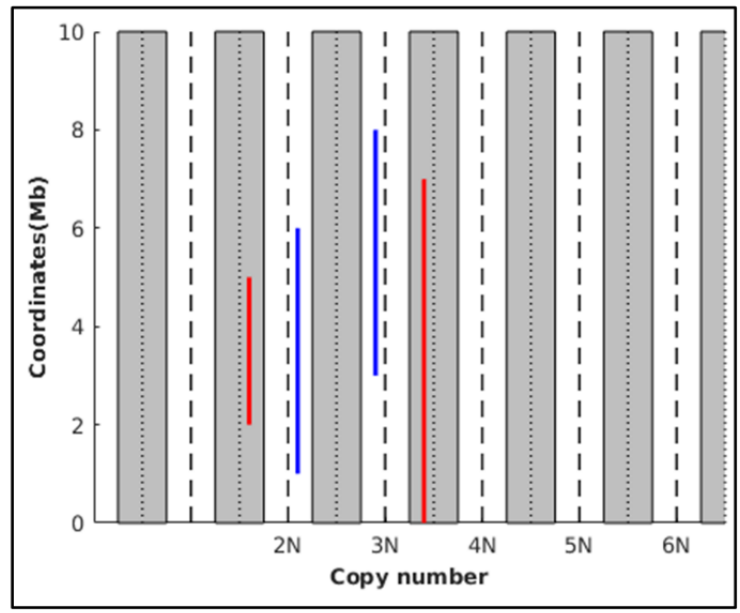

(c) Homogeneity score (HS) 


\section{Fig. 2}

Performance evaluation of AStra. (a) Dot plot showing the relationship of the homogeneity score with respect to the data coverage (left) and the ploidy levels (right). (b) Frequency distribution of VCaP cancer cell line (left: $10 \mathrm{~kb}$ bin; right: $100 \mathrm{~kb}$ bin) illustrating AStra can accurately identify the $\mathrm{CN}$ states at different resolutions. (c) Bar chart showing the number of sequencing datasets $(\mathrm{n}=26)$ identified by each model (M1-M10). (d) Comparison of centralization errors (CEs) under each model for 26 datasets. The number on the graph denotes the count of models whose CEs lie within $5 \%$ difference from the best (lowest) CE. 
(a)

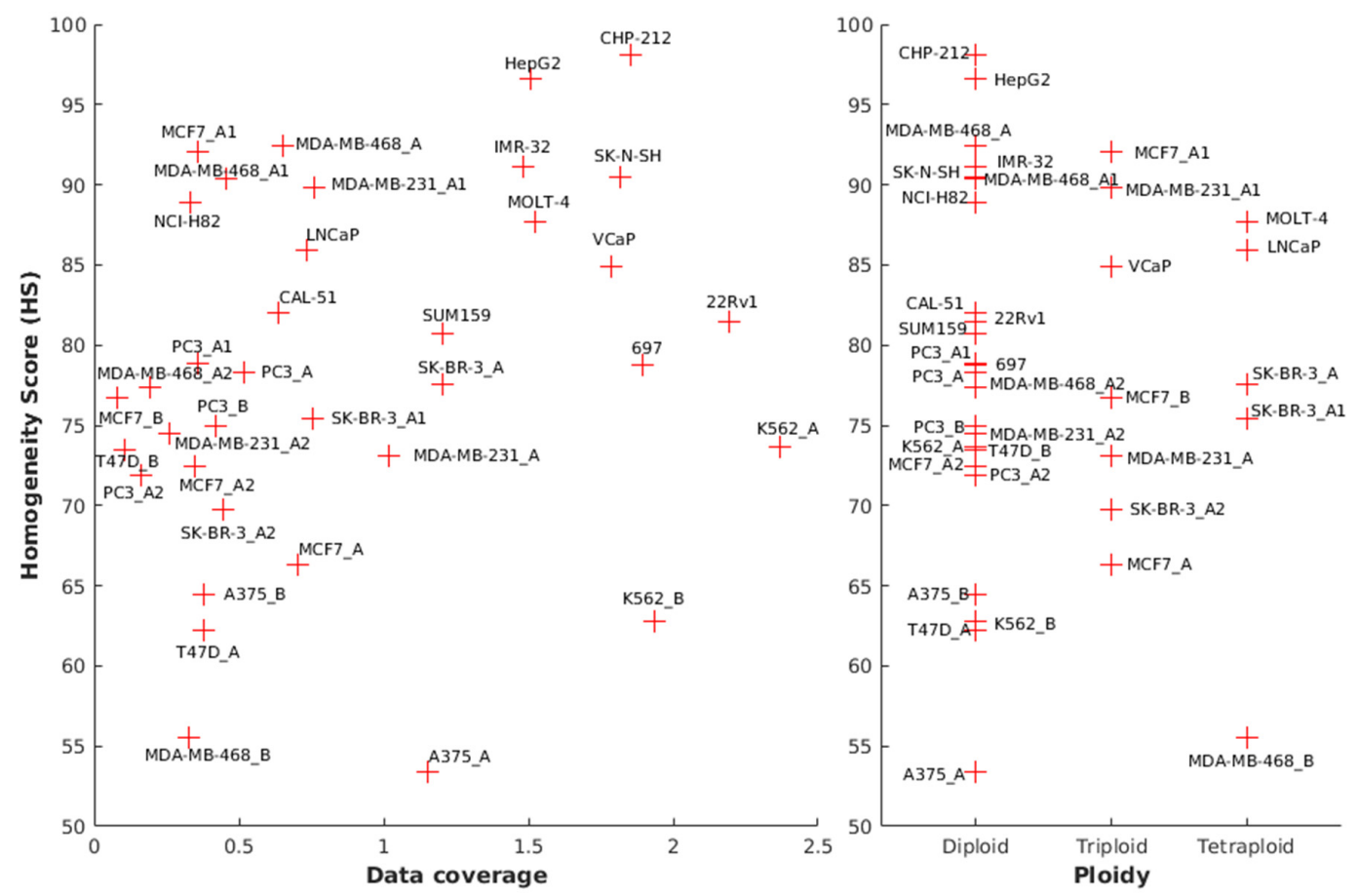

(b)
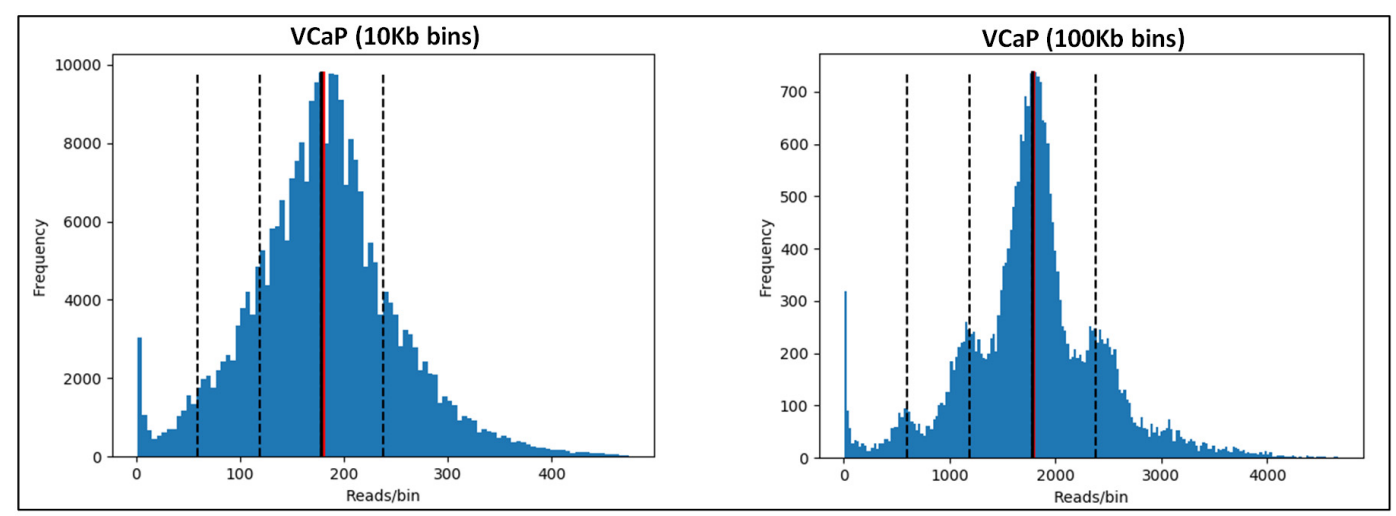

(c)

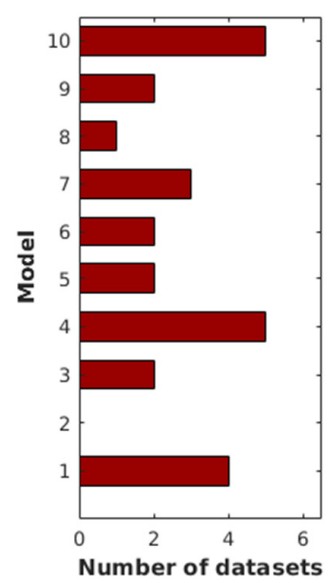

(d)

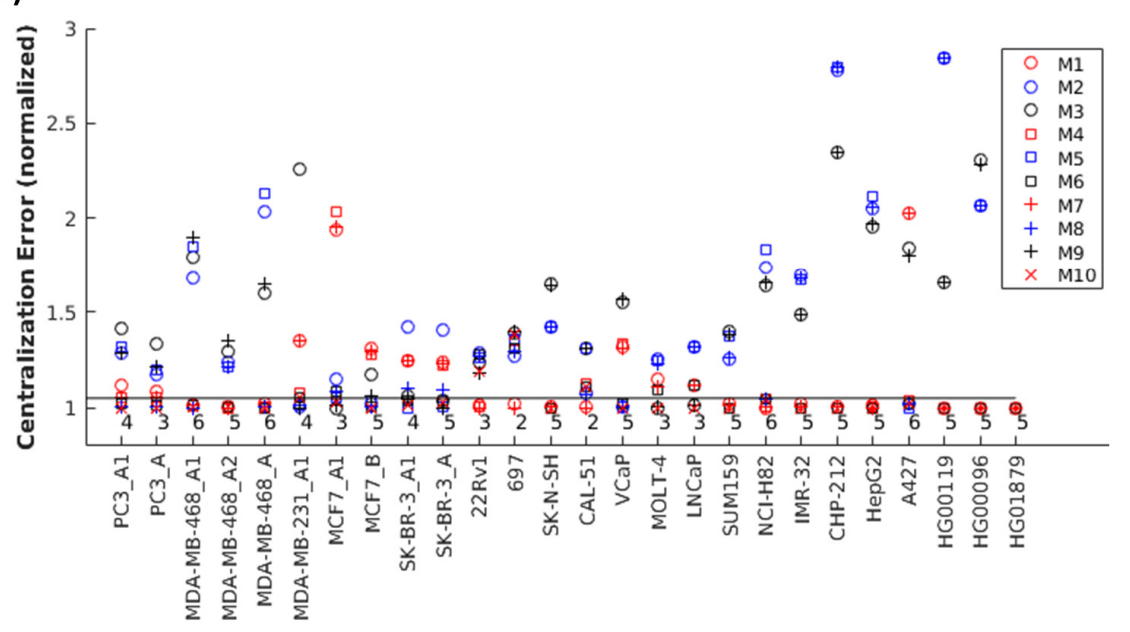




\section{Fig. 3}

Application of aneuploidy spectrum. (a) Bar chart showing the median errors of 26 sequencing datasets of different complexity and ploidy levels. For each dataset, the number on the graph is the estimated ploidy level. The blue bars represent 1000 genomes project samples while red bars represent cancer cell line data. (b) Visualization of the median error for MDA-MB-468_A (left) and LNCaP (right) cells. The black line denotes the ploidy state (major $\mathrm{CN}$ peak) whereas the red line denotes the global median of RD signal. The dotted black lines denote the other $\mathrm{CN}$ states. (c) Dot plot showing the relationship between the homogeneity score (HS) and the data coverage for 27 strains of MCF7. The area demarcated by blue line denotes a cluster of strains earlier reported to have similar single nucleotide variant cellular prevalence. (d) Comparison of doubling time (top) and the homogeneity score (bottom) of 27 strains of MCF7. 
(a)

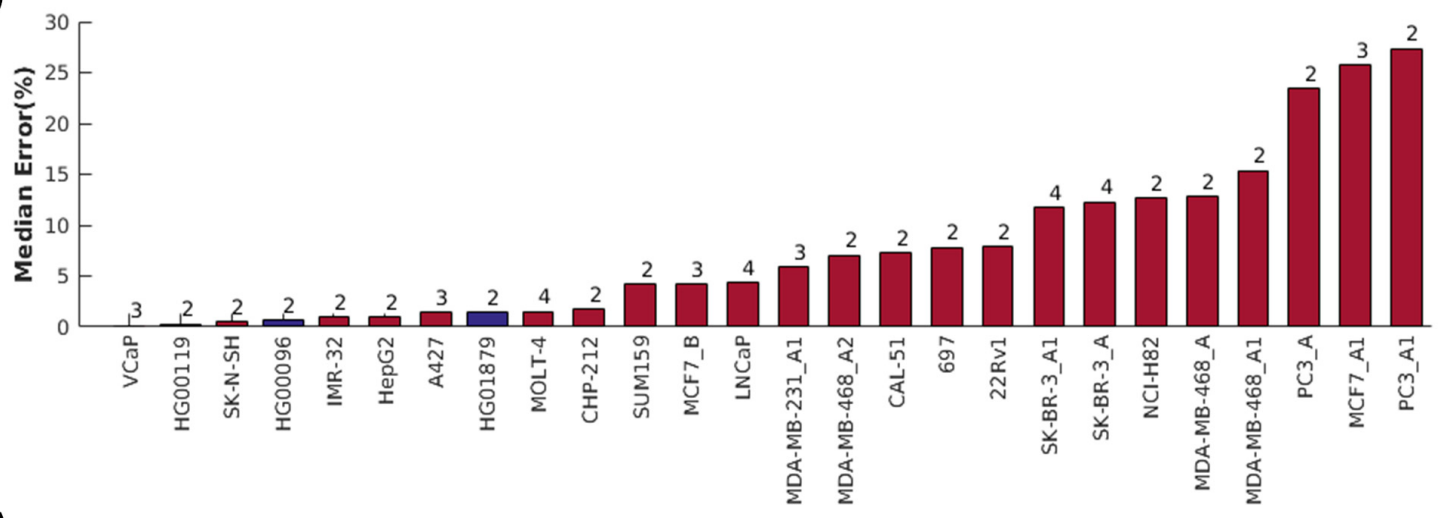

(b)

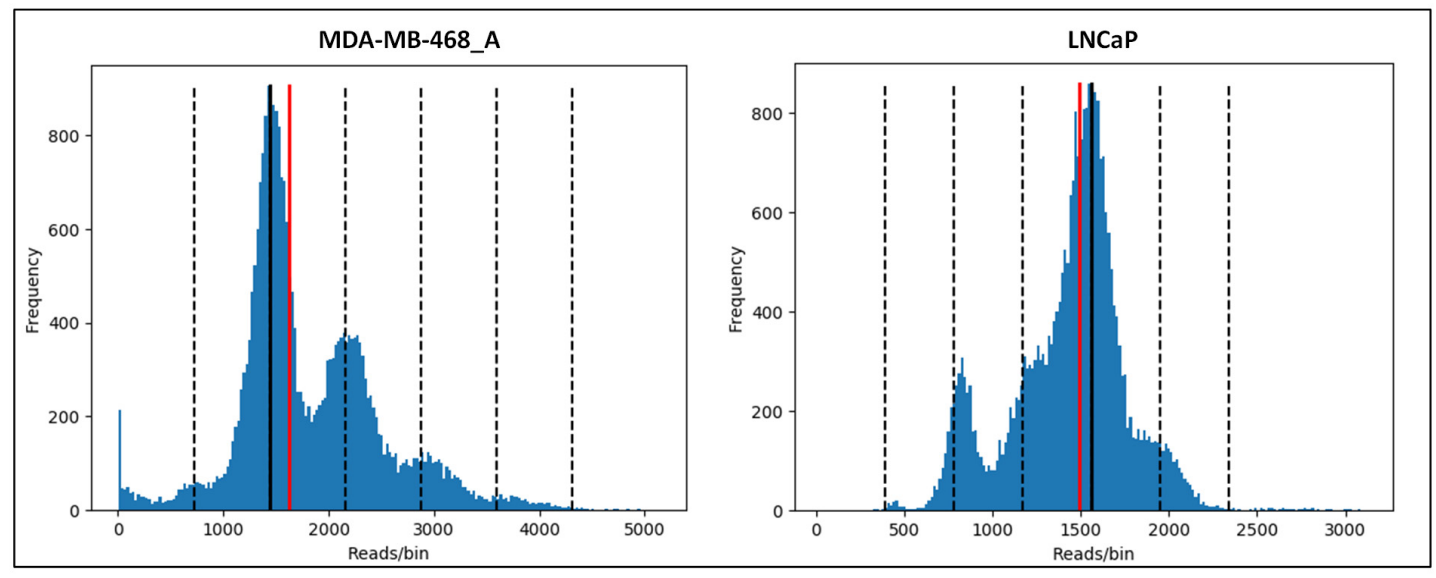

(c)

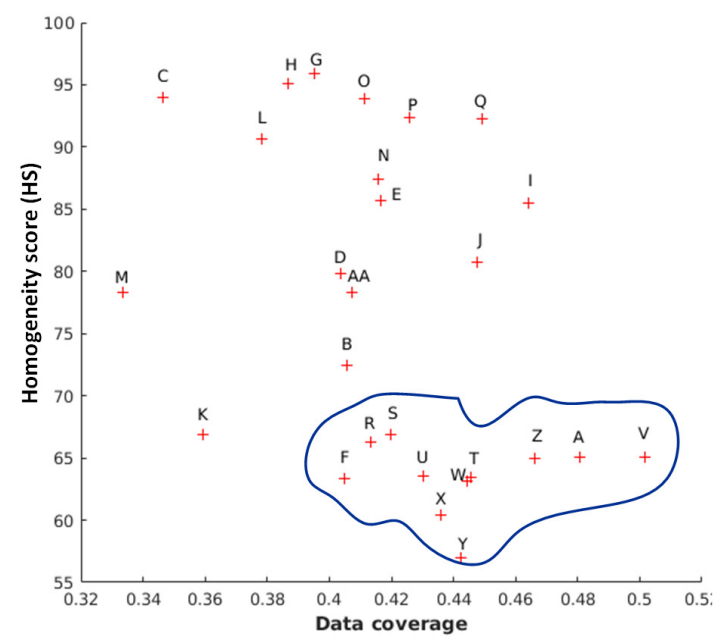

(d)
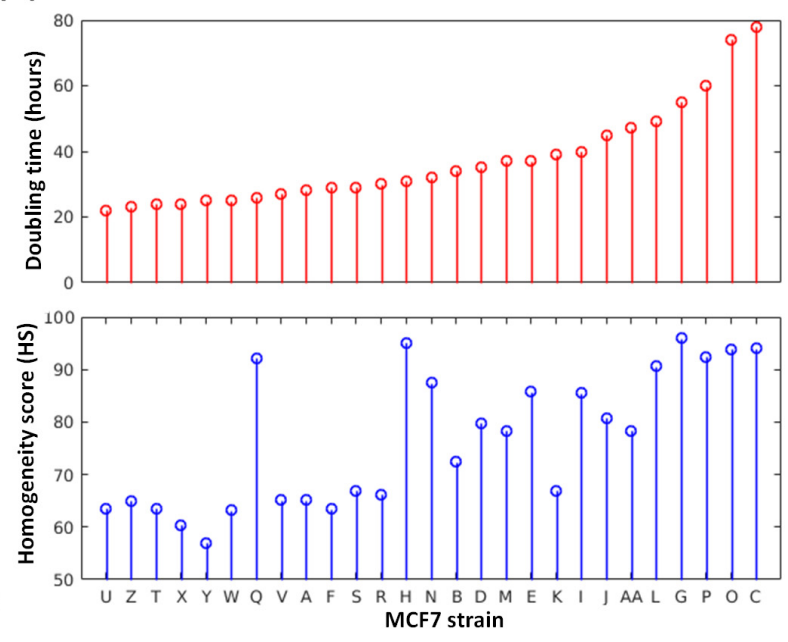